\title{
The effect of extraction, storage, and analysis techniques on the measurement of airborne endotoxin from a large dairy
}

\author{
Robert S. Dungan · April B. Leytem
}

Received: 5 May 2009/Accepted: 13 July 2009/Published online: 12 September 2009

(C) US Government 2009

\begin{abstract}
The objective of this study was to fill in additional knowledge gaps with respect to the extraction, storage, and analysis of airborne endotoxin, with a specific focus on samples from a dairy production facility. We utilized polycarbonate filters to collect total airborne endotoxins, sonication as the extraction technique, and $0.05 \%$ Tween 20 in pyrogen-free water (PFW) as the extraction solution. Endotoxin concentrations were determined via the Limulus amebocyte lysate (LAL) assay. The endotoxin concentrations in extracts after 15 and $30 \mathrm{~min}$ of filter sonication were similar, while the concentration in 60 min extracts was about twofold lower. Rapidly vortexing samples for up to 15 min after sonication did not increase the endotoxin concentration. However, concentrations were 13 and $26 \%$ lower in extracts that were centrifuged at 1,000 and $10,000 \mathrm{~g}$ for up to $15 \mathrm{~min}$, respectively. Field samples and endotoxin standard were also sonicated in glass or polypropylene tubes for up to $120 \mathrm{~min}$. Regardless of the extraction vessel, a decrease in endotoxin concentration occurred when sonicated for $>30 \mathrm{~min}$. Samples and endotoxin standard subjected to 12 freeze-thaw cycles at $-20^{\circ} \mathrm{C}$ only showed a slight
\end{abstract}

R. S. Dungan $(\square) \cdot$ A. B. Leytem

USDA-Agricultural Research Service (ARS), Northwest

Irrigation and Soils Research Laboratory, 3793 North

3600 East, Kimberly, ID 83341, USA

e-mail: robert.dungan@ars.usda.gov but not significant decrease in endotoxin concentration. Our results also demonstrate the importance of simultaneously adding LAL reagent to 96 -well plates before initiating the LAL assay.

Keywords Airborne - Bioaerosol .

Concentrated animal feeding operations .

Endotoxin - Limulus amebocyte lysate assay

\section{Introduction}

Due to high stocking densities at concentrated animal feeding operations, bioaerosols may be at sufficiently high levels to cause adverse health effects in both animals and humans (Millner 2009). Endotoxins, which are a major outer membrane constituent of Gram-negative bacteria, are a potent inducer of inflammatory reactions in the respiratory tract when inhaled. Bacterial endotoxins are lipopolysaccharide (LPS) molecules and the lipid A region of LPS is responsible for imparting the toxicity to the endotoxin (Bradley 1979). Exposure to airborne endotoxins can cause airway irritation, shortness of breath, chest tightness, cough, decreased lung function, and influenza-like symptoms (Rylander 2007). Chronic exposure to endotoxins in organic dusts from industrial and agricultural settings can lead to byssinosis (occupational lung disorder found in textile workers) 
and chronic bronchitis (Castellan et al. 1987; Jacobs 1989). On the other hand, environmental and occupational endotoxin exposures may protect against atopic sensitization and asthma in children and adults (Holla et al. 2002; Eduard et al. 2004; Portengen et al. 2005).

While the Limulus amebocyte lysate (LAL) assay is the most commonly used procedure to quantify airborne endotoxins (Milton et al. 1990), there is no universally agreed upon standard method for their collection, preparation, and analysis.

Only a few studies to date have investigated the effect of filter type, extraction solution, extraction method, transport conditions, and sample storage on the determination of airborne endotoxins from various residential, occupational, and agricultural settings (Walters et al. 1994; Douwes et al. 1995; Duchaine et al. 2001; Spaan et al. 2007). In environmental studies, the use of filters (e.g., glass, polycarbonate, cellulose ester, polyvinyl chloride, Teflon) is the preferred technique to capture airborne endotoxin (Clark et al. 1983; Dutkiewicz et al. 1989; Radon et al. 2002; Rao et al. 2007). Previous research has shown that two times more endotoxin can be extracted from polycarbonate, glass, and Teflon filters than from cellulose ester filters (Douwes et al. 1995). Endotoxins are extracted from the filters by sonicating or shaking them for up to $1 \mathrm{~h}$ in pyrogen-free water (PFW), buffered PFW, or Tween 20 in PFW. The extraction efficiency, when conducted using $0.05 \%$ Tween 20 in PFW, was shown to be seven times higher than that of PFW only (Douwes et al. 1995). Various transport conditions of dry filter samples (i.e., with and without desiccant) and storage of filter extracts at $4^{\circ} \mathrm{C}$ or $-20^{\circ} \mathrm{C}$ for up to $24 \mathrm{~h}$ did not affect endotoxin concentrations (Spaan et al. 2008).

The objective of this study was to fill in additional knowledge gaps with respect to the extraction, storage, and analysis of airborne endotoxins, with a specific focus on samples from a concentrated dairy production facility. The effect of the following variables on endotoxin concentrations was investigated in this study: extraction time, vortex time, centrifugation, extraction vessel (glass vs. polypropylene), and freeze-thaw. In addition, an experiment was performed to demonstrate the importance of simultaneously adding Kinetic-QCL reagent to a 96-well microplate before initiating the LAL assay.

\section{Materials and methods}

\subsection{Collection of total airborne endotoxins}

Total airborne endotoxins were collected from a 10,000 milking cow open-lot dairy in southern Idaho. The endotoxins were collected on $25 \mathrm{~mm} 1.0 \mu \mathrm{m}$ pore-size polycarbonate track-etched filters (Whatman, Florham Park, NJ, USA) that were housed in $25 \mathrm{~mm}$ open-face Delrin filter holders (Pall Corporation, East Hills, NY, USA). Except for the openface filter holders, all materials were depyrogenated by heating at $250^{\circ} \mathrm{C}$ for $30 \mathrm{~min}$ or purchased pyrogen-free. The open-face filter holders were depyrogenated by first rinsing with $70 \%$ ethanol, followed by a rinse with PFW, and then autoclaving for $1 \mathrm{~h}\left(1.23 \mathrm{~atm}\right.$ and $\left.121^{\circ} \mathrm{C}\right)$. The open-face filters were mounted on tripods at a height of $1.5 \mathrm{~m}$ and oriented into the wind at the downwind edge of the open lot. Vacuum was applied to the open-face filters using an SKC Vac-U-Go sampling pump (EightyFour, PA, USA). The sample collection time was $90 \mathrm{~min}$ at a rate of $2 \mathrm{~L} \mathrm{~min}^{-1}$.

Three open-face filters were mounted on each tripod and using a total of 9 tripods, 27 samples were collected simultaneously. Samples were collected at 0900 (morning), 1200 (noon), and 1500 (afternoon), for a total of 81 samples. The open-face filter holders, when not being used, were stored in pyrogen-free tins and transported in a cooler with ice packs. Trip blanks were prepared for each sampling event and were handled like the test filters, except that they were not exposed to ambient conditions. Upon reaching the laboratory, the polycarbonate filters were removed from the holders and placed into $2-\mathrm{mL}$ pyrogen-free polypropylene tubes and stored dry at $20^{\circ} \mathrm{C}$ until processed. Where applicable, the number of filters assigned to each of the experiments is indicated in the subsections below.

\subsection{Effect of Tween 20 and $\beta$-glucan blocker on calibration curves}

To determine the effect of Tween 20 and $\beta$-glucan blocker on calibration curves, endotoxin standards (lyophilized E. coli O55:B5; Lonza Inc., Walkersville, MD, USA) were prepared in PFW, PFW containing $0.05 \%(\mathrm{v} / \mathrm{v})$ Tween $20, \beta$-glucan blocker (Lonza Inc.), and $\beta$-glucan blocker containing $0.05 \%$ 
(v/v) Tween 20. An 8-point calibration curve ranging from 0.005 to 50 endotoxin units (EU) $\mathrm{mL}^{-1}$ was used.

\subsection{Effect of extraction vessel and sonication}

The purpose of this experiment was to assess the influence of polypropylene and borosilicate glass tubes on the recovery of endotoxins (field samples and calibration standards) during sonication periods of different length. Sonication is a commonly used technique to extract endotoxin from filters. For the field samples, a total of eight filters from the morning set were utilized (i.e., four replicates per treatment) and processed as described below. Pyrogen-free 2-mL polypropylene tubes were purchased from Thermo Fisher Scientific, Inc. (Waltham, MA, USA); pyrogen-free $10 \times 75 \mathrm{~mm}$ borosilicate glass tubes were purchased from Lonza Inc. Endotoxin calibration standards at $40 \mathrm{EU} \mathrm{mL}^{-1}$ were prepared in PFW and PFW containing 0.05\% (v/v) Tween 20 (PFW-Twn). Then, $1.5 \mathrm{~mL}$ of the calibration standard was added in quadruplicate to glass or polypropylene tubes. Dry polycarbonate filters were transferred into polypropylene and glass tubes, and $1.5 \mathrm{~mL}$ of either PFW or PFW-Twn was then added to the tubes. All treatments were initially sonicated for $15 \mathrm{~min}$ at $25^{\circ} \mathrm{C}$. Afterwards, the polycarbonate filters were removed from the tubes, and all treatments were sonicated for an additional 15, 30, or $60 \mathrm{~min}$. After each sonication period, aliquots were collected for analysis.

\subsection{Effect of sonication and vortexing}

This experiment was conducted to assess the effect of sonication time and vortex time on the quantity of endotoxin in the extraction solution when extracted from polycarbonate filters. Dry polycarbonate filters (18 from each collection time, for a total of 54 filters) were transferred into 2-mL polypropylene tubes, to which $1.5 \mathrm{~mL}$ of PFW-Twn was added. The filters were then sonicated for 15,30 , or $60 \mathrm{~min}$ (six filters per sonication treatment) at $25^{\circ} \mathrm{C}$, followed by the removal of the filters using depyrogenated forceps. Each of the six extracts per sonication treatment time was then vortexed for 1, 5, and $15 \mathrm{~min}$, with aliquots removed after each vortexing interval for analysis.

\subsection{Effect of centrifugation}

The purpose of this experiment was to determine the effect of centrifugation on the final concentration of endotoxin in the extraction solution. Morning samples (six replicates), which were extracted with PFWTwn by sonication and vortexing for $15 \mathrm{~min}$ each (from Table 1), were utilized for this experiment. After vortexing was completed, the extraction solution was centrifuged at 1,000 or $10,000 \mathrm{~g}$ for 1,5 , and $15 \mathrm{~min}$. An aliquot of the extract was removed before centrifugation and after each interval for analysis.

\subsection{Effect of freeze-thaw}

To assess the effect of multiple freeze-thaw cycles on endotoxin recovery, field samples (prepared in PFWTwn only) and a calibration standard of $40 \mathrm{EU} \mathrm{mL}^{-1}$ (prepared in PFW and PFW-Twn) were subjected to freezing at $-20^{\circ} \mathrm{C}$ and thawing at room temperature $\left(\sim 20^{\circ} \mathrm{C}\right)$. Endotoxin extracts from field samples collected in the morning (six replicates) and processed by sonicating for $30 \mathrm{~min}$ and vortexing for $15 \mathrm{~min}$ (from Table 1) were utilized for this experiment. All endotoxin samples were prepared in polycarbonate tubes, and aliquots were collected over the course of 12 freeze-thaw cycles. After each thaw cycle, the extracts were vortexed for $5 \mathrm{~min}$ prior to sample collection.

\subsection{Effect of delayed reagent addition}

The purpose of this experiment was to demonstrate that the addition of the Kinetic-QCL reagent to a 96-well microplate should be performed simultaneously. When using a single-channel or eight-channel pipette there is a delay in the addition of reagent as one works across the plate. We hypothesized that delaying the addition of reagent to the samples will cause samples that first received reagent to have elevated concentrations. A 96-channel pipette (Transtar-96 system, Costar, Cambridge, MA, USA) was used for simultaneous addition of the reagent to the wells, while an eight-channel pipette (Eppendorf, Westbury, NY, USA) was used to demonstrate the effect of delayed addition. Six replicate field samples were extracted by sonicating in PFW-Twn for $30 \mathrm{~min}$ at $25^{\circ} \mathrm{C}$. The replicates were placed in eight columns of a 96-well microplate, upon which Kinetic-QCL reagent was added to four of the columns using an 8-channel 
Table 1 Effect of sonication and vortexing on endotoxin concentrations in dairy samples

\begin{tabular}{lrlrl}
\hline Filter sonication $(\mathrm{min})$ & Extract vortexing $(\mathrm{min})$ & \multicolumn{3}{l}{ Concentration $\left(\mathrm{EU} \mathrm{mL}^{-1}\right)$} \\
\cline { 3 - 5 } & & Morning & Noon & Afternoon \\
\hline \multirow{2}{*}{15} & 1 & $37.2(3.1)^{\mathrm{a}}$ & $16.5(2.6)$ & $20.7(2.5)$ \\
& 5 & $40.2(2.1)$ & $19.2(2.8)$ & $19.5(1.2)$ \\
& 15 & $43.8(3.3)$ & $17.4(2.7)$ & $20.3(2.5)$ \\
30 & 1 & $28.8(3.5)$ & $16.5(2.9)$ & $12.8(2.3)$ \\
& 5 & $47.1(5.1)$ & $19.2(2.9)$ & $14.9(2.7)$ \\
& 15 & $42.3(5.2)$ & $18.7(3.4)$ & $13.2(1.6)$ \\
60 & 1 & $21.9(4.9)$ & $7.6(0.9)$ & $6.8(1.4)$ \\
& 5 & $16.5(2.2)$ & $6.0(1.1)$ & $6.4(1.4)$ \\
& 15 & $17.6(2.1)$ & $7.8(1.3)$ & $6.4(1.5)$ \\
\hline
\end{tabular}

Six filters were utilized for each sonication event and collection time, for a total of 54 filters

a $\mathrm{SE}$ of the mean $(n=6)$ microplate reader (BioTek Instruments, Inc., Winooski, VT, USA) to initiate the test. The $\mathrm{pH}$ of the samples, when combined with the reagent, was 7.5. To ensure quality control and assurance, trip blanks and duplicate samples were run regularly.

\subsection{Statistical analyses}

Endotoxin concentrations were tested for normality using the Shapiro-Wilk test with the PROC CAPABILITY procedure of SAS (SAS Institute Inc. 2004). The data were analyzed using the Mixed Models procedure of SAS. The data from experiments in Sect. 2.4 were analyzed with sonication time, vortex time, and their interaction as fixed effects and time of day as a random effect. The data from Sect. 2.5 were analyzed with centrifugal force, time, and their interaction as fixed effects (subsequently the model was run again with time as a fixed effect at each centrifugal force level). The data from Sect. 2.6 were analyzed with sample (standard in PFW-Twn, standard in PFW, field samples), freeze-thaw cycle, and their interaction as fixed effects. Means separation was carried out using the difference of the least squares means with Tukey-Kramer adjustment and $\alpha=0.10$. Statements of statistical significance were based upon $P<0.10$ unless otherwise stated. Linear regression modeling was performed using SigmaPlot 11.0 (Systat Software Inc., Chicago, IL, USA).

\section{Results and discussion}

PFW-Twn has received considerable interest as an endotoxin extraction solution since it has been shown 
to increase the extraction efficiency by as much as sevenfold over that of PFW only (Douwes et al. 1995; Spaan et al. 2007). Figure 1 shows an endotoxin calibration curve, where the primary and secondary standards were prepared in either PFW or PFW with $0.05 \%$ Tween 20 . The linear regression curves (all with $\left.r^{2}=0.99\right)$ somewhat parallel each other, but converge at the lowest endotoxin concentration, and the PFW-Twn standards shift toward slightly higher $\Delta t$ onset (i.e., time at which the absorbance increases 0.2 units). In another experiment, the same shift occurred when the PFW contained up to 10 times less Tween 20 (data not shown). This shift in the standard curve was also observed by Spaan et al. (2008) when endotoxin standards were prepared in PFW-Twn. The probable reason for this outcome is that the Tween is reducing the activity of the endotoxin and/or proenzyme in the LAL; thus, there is a delay in $\Delta t$ onset. Based on our calibration data, when endotoxin samples are extracted in PFW-Twn and concentrations are determined with calibration standards prepared in PFW only, samples will have lower than expected concentrations. For example, a sample extracted with PFW-Twn that has an endotoxin concentration of $40 \mathrm{EU} \mathrm{mL}{ }^{-1}$, would only calculate out to have a concentration of about $8.2 \mathrm{EU} \mathrm{mL}^{-1}$ if the standards were prepared in PFW. Therefore, it is essential that the calibration standards be prepared in PFW-Twn if the extraction solution contains Tween. Because $\beta$-1,3-glucans have been shown to inhibit or enhance the LAL reaction (Morita et al. 1981; Roslansky and Novitsky 1991; Milton et al. 1997), we also prepared calibration standards with $\beta$-glucan blocker or $\beta$-glucan blocker with $0.05 \%$ Tween 20 . According to the manufacturer's protocol (Lonza Inc.), samples should be diluted 1:1 with $\beta$-glucan blocker before being analyzed. Similarly, the calibration standards containing Tween shifted away from those without Tween (Fig. 1). Because the $\beta$-glucan blocker did not interfere with the calibration, it is not necessary to prepare the standards with $\beta$-glucan blocker if it is being used in the assay.

To assist in the extraction of endotoxin from filter samples, sonication or shaking in pyrogen-free polypropylene or glass vessels has been utilized (Clark et al. 1983; Chang et al. 2001; Duchaine et al. 2001; Rao et al. 2007). Sonication was chosen as the extraction technique in this study, which has been shown to be just as effective as direct methanolysis of

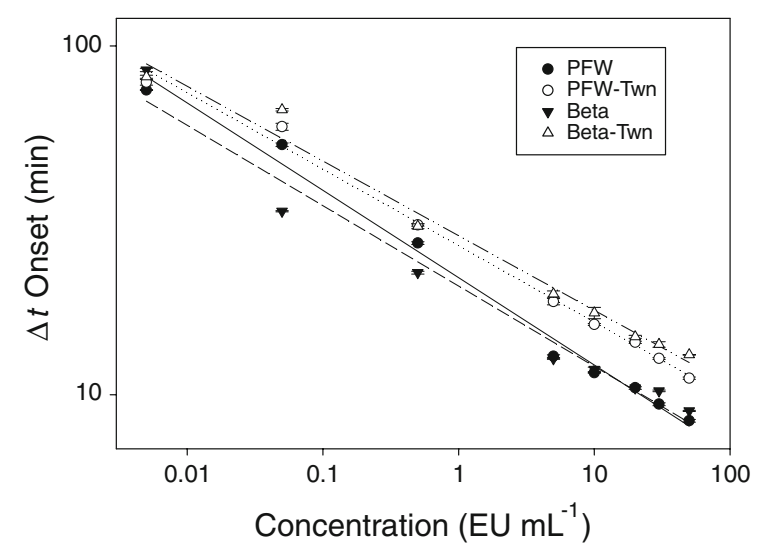

Fig. 1 Calibration curves of endotoxin standards prepared in PFW, PFW-Twn, Beta, and Beta-Twn. Error bars represent the SE of the mean $(n=3)$. PFW, pyrogen-free water; Twn, Tween at $0.05 \%$; Beta, $\beta$-glucan blocker

samples followed by GC-MS analysis (Walters et al. 1994). While borosilicate glass tubes appear to be used more often than polypropylene tubes in the literature, there is little evidence suggesting that pyrogen-free polypropylene tubes cannot be effectively utilized for extracting and storing endotoxin samples. Novitsky et al. (1986), however, recommended that some lots of this polymer should be avoided as they can irreversibly adsorb large amounts of LPS. Endotoxins are also adsorbed by glass, as pointed out by Novitsky et al. (1986) in the LAL Kinetic-QCL protocol. To counter the adsorption to glass, vigorous vortexing of endotoxin standard for $15 \mathrm{~min}$ is recommended.

In this study, field samples and endotoxin standard (prepared in PFW and/or PFW-Twn) were placed in either polypropylene or borosilicate glass tubes and sonicated for up to $120 \mathrm{~min}$ to test the effect on endotoxin recovery. The data were normalized at the 15-min point, as this is when the filters were removed from the field samples (Fig. 2). Sonication of the samples for an additional $15 \mathrm{~min}$ (i.e., total time of 30 min) reveals a slight decline in the endotoxin concentration, except in the field sample extracted in PFW-Twn in glass. At 60 and $120 \mathrm{~min}$ of total sonication time, there was a considerable decline in endotoxin concentrations in the treatments. However, the endotoxin concentration in the field sample prepared in the glass tubes was up to 4.5 times higher than the other treatments. Interestingly, this same effect was not seen when endotoxin standard was 


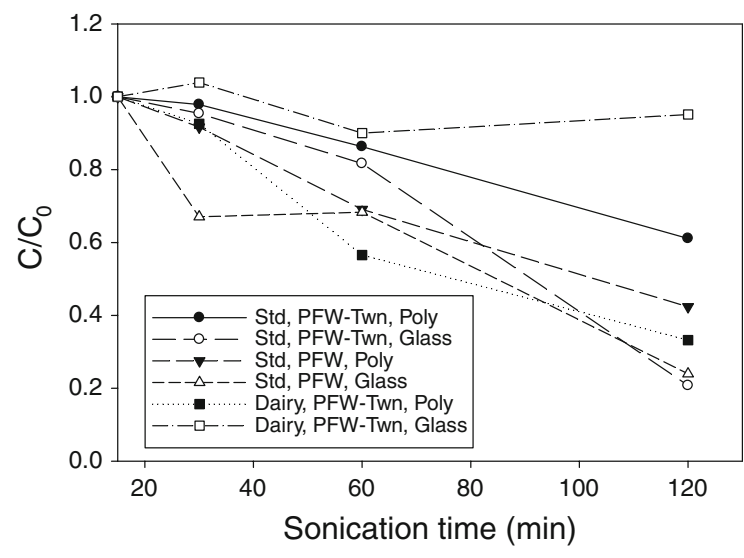

Fig. 2 Effect of sonication time and extraction vessel (polypropylene and borosilicate glass) on the recovery of endotoxin from a calibration standard and dairy samples prepared in PFW and/or PFW-Twn. A total of eight filter samples from the morning set were utilized; data were generated using four replicates per treatment. $\mathrm{C} / \mathrm{Co}$, concentration/initial concentration; PFW, pyrogen-free water; Twn, Tween at $0.05 \%$

prepared with PFW or PFW-Twn in glass. Overall, it appears that the loss in endotoxin activity is not due to the extraction vessel material, but is a result of endotoxin deactivation. Sonication, which is often used to disrupt cellular membranes and deactivate biological materials, may be the cause of this outcome.

Table 1 shows endotoxin data from field samples that were collected at three separate times during the day at the open-lot dairy. The filters were removed after 15,30 , or $60 \mathrm{~min}$ of sonication, and the extracts were subsequently vortexed for 1,5 , or $15 \mathrm{~min}$ before being analyzed. When a Mixed Models procedure of SAS was applied to the data, there was no significant effect of vortexing and no significant interaction between vortexing and sonication $(\alpha=0.10)$. The effect of sonication time was significant $(\alpha=0.10)$, as sonication time increased endotoxin concentrations decreased. This supports our data above, where sonication longer than 30 min substantially reduced endotoxin recovery.

A few studies have indicated that endotoxin extracts were centrifuged prior to analysis via the LAL assay (Olenchock et al. 1989; Douwes et al. 1995; Madsen 2006; Spaan et al. 2007). While one could justify this as a means to remove inhibitory and enhancing substances, we could not find a protocol in the literature that recommends centrifugation of endotoxin extracts or explains its purpose. Substances in air samples have been shown to inhibit or enhance the LAL assay (Hollander et al. 1993; Milton et al. 1997). Although not addressed in this study, the use of a resistant-parallel-line estimation has been recommended, where the analysis of dose-response curves is used to reduce the potential for interference and to increase the precision of the LAL assay (Milton et al. 1990). If the endotoxins are attached to particles that are of greater mass than the endotoxins, then the endotoxin concentration should theoretically be lower after centrifugation. Figure 3 shows the effect of centrifugation at 1,000 and $10,000 \mathrm{~g}$ on the endotoxin concentration in extracts from field samples. There was a significant main effect of centrifuge time on endotoxin concentration, while the centrifugal force and interaction terms were not significant $(\alpha=0.10)$. After $1 \mathrm{~min}$ of centrifugation, the concentrations were reduced by approximately 13 and $27 \%$ at 1,000 and $10,000 \mathrm{~g}$, respectively. Our results indicate that some of the airborne endotoxins were associated with larger particles (e.g., dust); however, the possibility existed that the endotoxins were not being removed by centrifugation, but substances that enhance the LAL reaction were being removed instead. To verify whether enhancing substances were present in the extracts, we diluted samples up to 800 times in PFWTwn. No loss of endotoxin activity was noted in the dilution series (data not shown).

The effect of repeated freeze-thaw cycles on endotoxin recovery from calibration standards and field samples is shown in Fig. 4. When up to 12 freeze-thaw cycles at $-20^{\circ} \mathrm{C}$ were performed, as much as $96 \%$ of the endotoxin was recovered from the field sample extract, and 97 and $91 \%$ was recovered from the calibration standards that were prepared in PFW-Twn or PFW, respectively. There was no significant main effect of sample, freeze-thaw cycle, or their interaction $(\alpha=0.10)$. Our results contradict those obtained by Douwes et al. (1995), who found up to a $90 \%$ reduction in endotoxin concentrations when a commercially available standard (prepared in PFW) was subjected to eight freeze-thaw cycles and stored in polypropylene and glass (borosilicate and "soft") containers. A similar effect was also noted with endotoxins measured in house dust extracts. Endotoxin standard stored at $20^{\circ} \mathrm{C}$ in polypropylene and glass for 1 year and not subjected to freeze-thaw cycles, however, did not show a significant decrease in endotoxin concentration (Douwes et al. 1995). Milton et al. (1997) stored 


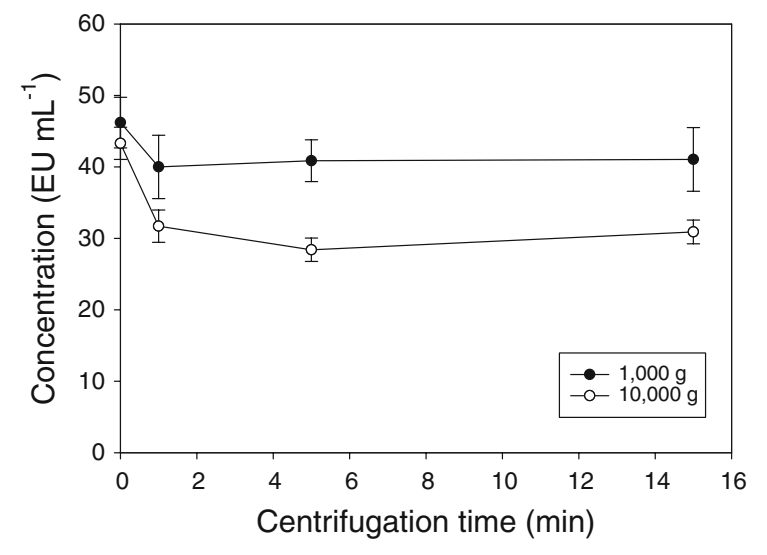

Fig. 3 Effect of centrifugation on the recovery of endotoxin in dairy samples extracted in PFW-Twn. Error bars represent the $\mathrm{SE}$ of the mean $(n=6)$. PFW, pyrogen-free water; Twn, Tween at $0.05 \%$

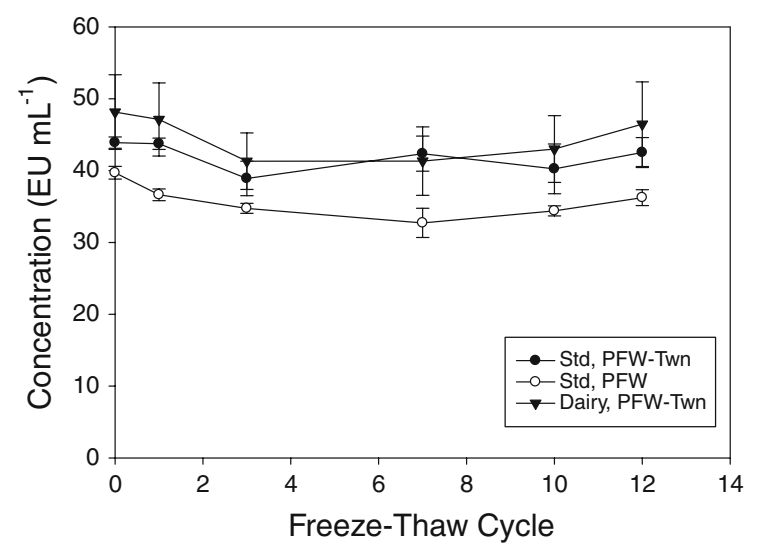

Fig. 4 Effect of freeze-thaw cycles $\left(-20^{\circ} \mathrm{C}\right)$ on the recovery of endotoxin from calibration standards and dairy samples prepared in PFW or PFW-Twn. Error bars represent the SE of the mean $(n=6)$. PFW, pyrogen-free water; Twn, Tween at $0.05 \%$

buffer-extracted endotoxins from house dust at $20^{\circ} \mathrm{C}$ for up to 6 weeks in glass and showed an $86 \%$ decline in the endotoxin concentration. In a freezethaw experiment at $-85^{\circ} \mathrm{C}$ with water extracts of airborne grain dusts, the endotoxin concentration was not significantly affected after 13 cycles (Olenchock et al. 1989). While our freeze-thaw results are more in line with those obtained by Olenchock et al. (1989), the results observed by Douwes et al. (1995) may be linked to the endotoxin source and its susceptibility to freezing and thawing. Irreversible adsorption of endotoxin to the container material was

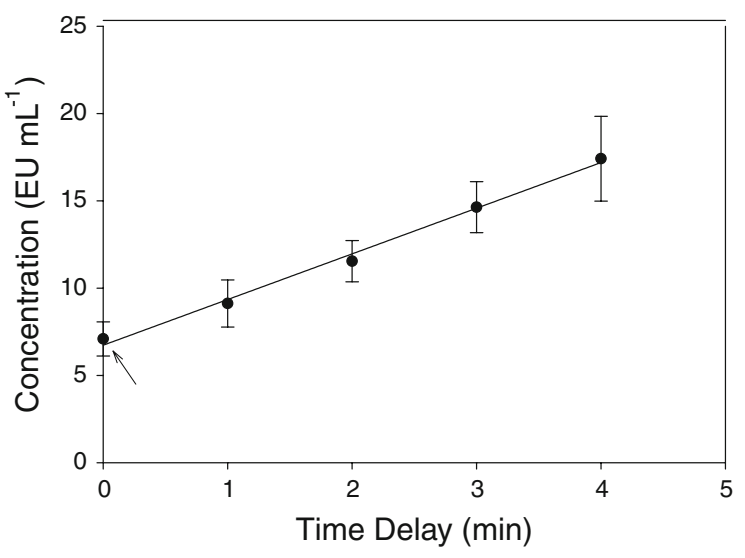

Fig. 5 Effect of time delay on the addition of LAL reagent to a 96-well microplate. Error bars represent the SE of the mean $(n=6)$. The arrow indicates samples that received reagent using the 96-channel pipette $(n=24)$

ruled out although it has been shown to occur with both polypropylene and glass (Novitsky et al. 1986). This is clearly an area that deserves further attention.

The manufacturer of the LAL Kinetic-QCL test kit recommends the use of an eight-channel pipette to dispense $100 \mu \mathrm{L}$ aliquots of Kinetic-QCL reagent into the wells of the microplate. During preliminary studies, we found that duplicate samples placed on the far end of the microplate were consistently higher, sometimes by as much as twofold. This prompted us to conduct an experiment, where we intentionally delayed the addition of reagent between columns of identical samples. Our results in Fig. 5 show that for every 1 min delay in reagent addition, samples will produce a linear increase in concentration $\left(r^{2}=0.99\right)$. After a 4 min delay, the endotoxin concentration was 2.5-fold higher than when the reagent was added to the standards and samples (four columns) simultaneously using a 96-channel pipette and immediately placed in the plate reader (i.e., time zero samples). The manufacturer's protocol does advise adding the reagent as quickly as possible, but from our personal experience, filling 12 columns of a 96-well microplate with an eight-channel pipette takes approximately 4 min (more or less depending upon the technician). This also demonstrates the importance of placing the microplate in the plate reader immediately following reagent addition. Any delay between reagent addition and placement of the microplate in the plate reader will likely result in an overestimation of endotoxin concentration. 


\section{Conclusions}

Previous research has demonstrated that endotoxin extraction efficiencies from filters are greatest when Tween is used in the extraction solution (Douwes et al. 1995; Spaan et al. 2007). Based on the airborne endotoxins collected in this study, the greatest extraction efficiency from polycarbonate filters occurred when they were sonicated for 15-30 min. However, to avoid deactivating the endotoxins, polycarbonate filters extracted using PFW-Twn should not be sonicated for more than $30 \mathrm{~min}$. Shaking is an alternative extraction technique that was not investigated in this study, but should also receive consideration. Vortexing the extracts after sonication did not result in higher endotoxin concentrations, but it may be necessary to improve the recovery of endotoxins from certain sample types not addressed in this study. While borosilicate glass is the recommended extraction vessel material, our results do demonstrate that polypropylene can also be successfully used. Since both glass and polypropylene are known to adsorb endotoxin, it is always recommended that stored extracts and standards be vortexed before an aliquot is removed for analysis. Our results also suggest that endotoxin extracts can be stored frozen at $-20^{\circ} \mathrm{C}$ and subjected to multiple freeze-thaw cycles, with little or no effect on the final endotoxin concentration. To avoid analytical errors with the LAL assay, calibration standards should be prepared in the same matrix as the extraction solution (e.g., PFW-Twn), or an underestimation of the endotoxin concentration will occur. If the extracts are prepared with $\beta$-glucan blocker, it is not necessary to prepare the calibration standards with $\beta$-glucan blocker. Additional analytical errors will occur if the Kinetic QCL-reagent is dispensed into a 96-well microplate using an eight-channel pipette, especially if multiple columns are being used, and there is delay getting the microplate to the reader. We highly recommend the use of a 96-channel pipette so that all samples are treated simultaneously.

\section{References}

Bradley, S. G. (1979). Cellular and molecular mechanisms of action of bacterial endotoxins. Annual review of microbiology, 33, 67-94.
Castellan, R. M., Olenchock, S. A., Kinsley, K. B., \& Hankinson, J. L. (1987). Inhaled endotoxin and decreased spirometric values. The New England journal of medicine, 317, 605-610.

Chang, C. W., Chung, H., Huang, C. F., \& Su, H. J. J. (2001). Exposure of workers to airborne microorganisms in openair swine houses. Applied and Environmental Microbiology, 67, 155-161.

Clark, C. S., Rylander, R., \& Larsson, L. (1983). Levels of gram-negative bacteria, Aspergillus fumigatus, dust, and endotoxin at compost plants. Applied and Environmental Microbiology, 45, 1501-1505.

Douwes, J., Versloot, P., Hollander, A., Heederik, D., \& Doekes, G. (1995). Influence of various dust sampling and extraction methods on the measurement of airborne endotoxin. Applied and Environmental Microbiology, 61, 1763-1769.

Duchaine, C., Thorne, P. S., Meriaux, A., Grimard, Y., Whitten, P., \& Cormier, Y. (2001). Comparison of endotoxin exposure assessment by bioaerosol impinger and filtersampling methods. Applied and Environmental Microbiology, 67, 2775-2780.

Dutkiewicz, J., Olenchock, S. A., Sorenson, W. G., Gerencser, V. F., May, J. J., Pratt, D. S., et al. (1989). Levels of bacteria, fungi, and endotoxin in bulk and aerosolized corn silage. Applied and Environmental Microbiology, 55, 1093-1099.

Eduard, W., Douwes, J., Omenass, E., \& Heederik, D. (2004). Do farming exposures cause or prevent asthma? Results from a study of adult Norwegian farmers. Thorax, 59, 381-386.

Holla, A. D., Roy, S. R., \& Liu, A. H. (2002). Endotoxin, atopy and asthma. Current opinion in Allergy and Clinical Immunology, 2, 141-145.

Hollander, A., Heederik, D., Versloot, P., \& Douwes, J. (1993). Inhibition and enhancement in the analysis of airborne endotoxin levels in various occupational environments. American Industrial Hygiene Association Journal, 54, 647-653.

Jacobs, R. R. (1989). Airborne endotoxins: An association with occupational lung disease. Applied Industrial Hygiene, 4, 50-56.

Madsen, A. M. (2006). Airborne endotoxin in different background environments and seasons. Annals of Agricultural and Environmental Medicine, 13, 81-86.

Millner, P. D. (2009). Bioaerosols associated with animal production operations. Bioresource Technology, 100, 5379-5385.

Milton, D. K., Gere, R. J., Feldman, H. A., \& Greaves, I. A. (1990). Endotoxin measurement: Aerosol sampling and application of a new Limulus method. American Industrial Hygiene Association Journal, 51, 331-337.

Milton, D. K., Johnson, D. K., \& Park, J.-H. (1997). Environmental endotoxin measurement: Interference and sources of variation in the Limulus assay of house dust. American Industrial Hygiene Association Journal, 58, 861-867.

Morita, T., Tanaka, S., Nakamura, T., \& Iwanaga, S. (1981). A new $(1 \rightarrow 3)$ - $\beta$-D-glucan-mediated coagulation pathway found in Limulus amebocytes. FEBS Letters, 129, 319-321. 
Novitsky, T. J., Schmidt-Gengenbach, J., \& Remillar, J. F. (1986). Factors affecting the recovery of endotoxin adsorbed to container surfaces. Journal of Parenteral Science and Technology, 40, 284-286.

Olenchock, S. A., Lewis, D. M., \& Mull, J. C. (1989). Effects of different extraction protocols on endotoxin analysis of airborne grain dust. Scandinavian Journal of Work, Environment and Health, 15, 430-435.

Portengen, L., Preller, L., Tielen, M., Doekes, G., \& Heederik, D. (2005). Endotoxin exposure and atopic sensitization in adult pig farmers. The Journal of Allergy and Clinical Immunology, 115, 797-802.

Radon, K., Danuser, B., Iversen, M., Monso, E., Weber, C., Hartung, J., et al. (2002). Air contaminants in different European farming environments. Annals of Agricultural and Environmental Medicine, 9, 41-48.

Rao, C. Y., Riggs, M. A., Chew, G. L., Muilenberg, M. L., Thorne, P. S., Van Sickle, D., et al. (2007). Characterization of airborne molds, endotoxins, and glucans in homes in New Orleans after hurricanes Katrina and Rita. Applied and Environmental Microbiology, 73, 1630-1634.
Roslansky, P. F., \& Novitsky, T. J. (1991). Sensitivity of Limulus amebocyte lysate (LAL) to LAL-reactive glucans. Journal of Clinical Microbiology, 29, 2477-2483.

Rylander, R. (2007). Endotoxin in the air: Good or bad for you? Clinical Pulmonary Medicine, 14, 140-147.

SAS Institute Inc. (2004). SAS/STAT software, Version 9.2. Cary, NC, USA.

Spaan, S., Doekes, G., Heederik, D., Thorne, P. S., \& Wouters, I. M. (2008). Effect of extraction and assay media on analysis of airborne endotoxin. Applied and Environmental Microbiology, 74, 3804-3811.

Spaan, S., Heederik, D. J. J., Thorne, P. S., \& Wouters, I. M. (2007). Optimization of airborne endotoxin exposure assessment: Effects of filter type, transport conditions, extraction solutions, and storage of samples and extracts. Applied and Environmental Microbiology, 73, 6134-6143.

Walters, M., Milton, D., Larsson, L., \& Ford, T. (1994). Airborne environmental endotoxin: A cross-validation of sampling and analysis techniques. Applied and Environmental Microbiology, 60, 996-1005. 